\title{
Efficient, Physiologically Realistic Lung Airflow Simulations
}

\author{
D. Keith Walters*, Greg W. Burgreen, David M. Lavallee, David S. Thompson, and Robert L. Hester
}

\begin{abstract}
One of the key challenges for computational fluid dynamics (CFD) simulations of human lung airflow is the sheer size and complexity of the complete, multiscale geometry of the bronchopulmonary tree. Since 3-D CFD simulations of the full airway tree are currently intractable, researchers have proposed reduced geometry models in which multiple airway paths are truncated downstream of the first few generations. This paper investigates a recently proposed method for closing the CFD model by application of physiologically correct boundary conditions at truncated outlets. A realistic, reduced geometry model of the lung airway based on CT data has been constructed up to generation 18, including extrathoracic, bronchi, and bronchiole regions. Results indicate that the new method yields reasonable results for pressure drop through the airway, at a small fraction of the cost of fully resolved simulations.
\end{abstract}

Index Terms-Computational fluid dynamics (CFD), lung morphology, numerical methods, respiration.

\section{INTRODUCTION}

A CCURATE knowledge of airflow and particle deposition is critically important for understanding the health impacts of inhaled particulates in humans. These include potentially harmful substances such as environmental pollutants as well as aerosolized medicines used in pulmonary drug delivery. The ability to efficiently model or simulate the airflow in the lung would provide a valuable alternative to costly and potentially harmful in vivo experimental methods.

Predicting airflow in the lung airway is complicated by several issues, including limitations of existing imaging technologies, patient specific variations in lung morphology, and changes in airway geometry during the breathing cycle, as well as the extreme size and multiscale nature of the airway geometry [1]. With regard to the latter, the overall airway includes

Manuscript received April 2, 2011; revised May 24, 2011; accepted June 20, 2011. Date of publication July 14, 2011; date of current version September 21, 2011. This work was supported in part by the U.S. National Science Foundation under Grant EPS-0903787. Asterisk indicates corresponding author.

*D. K. Walters is with the Department of Mechanical Engineering, Mississippi State University, Mississippi State, MS 39762 USA (e-mail: walters@me.msstate.edu).

G. W. Burgreen is with the Center for Advanced Vehicular Systems, Mississippi State University, Mississippi State, MS 39762 USA (e-mail: greg.burgreen@msstate.edu).

D. M. Lavallee and D. S. Thompson are with the Department of Aerospace Engineering, Mississippi State University, Mississippi State, MS 39762 USA (e-mail:dml148@msstate.edu; dst@ae.msstate.edu).

R. L. Hester is with the Department of Physiology, University of Mississippi Medical Center, Jackson, MS 39216 USA (e-mail: rhester@umc.edu).

Color versions of one or more of the figures in this paper are available online at http://ieeexplore.ieee.org.

Digital Object Identifier 10.1109/TBME.2011.2161868 extrathoracic regions (mouth and trachea), the conducting region (bronchi and bronchioles), and the alveolated pulmonary region. The airway tree is made up of successive bifurcating segments, comprised of approximately 23-25 total generations, including approximately 16-18 in the bronchial region (conducting zone). The resulting geometry contains on the order of $10 \mathrm{M}$ flow segments with diameters ranging from approximately $2 \mathrm{~cm}$ (trachea) to $400 \mu \mathrm{m}$ [2].

Most previous computational fluid dynamics (CFD) studies of the lung airway have focused on small subsections, for example, single [3] or triple [4]-[6] bifurcations. A few notable exceptions have addressed the large, multiscale geometry of the entire tracheobronchial tree. These include the sequential simulations of Nowak et al. [7] and Zhang et al. [8], the hybrid CFD/1-D model of Ma and Lutchen [9], and the partially resolved multigeneration models of Gemci et al. [10] and De Backer et al. [11]. Recently, Tian et al. [12] have proposed a hybrid model composed of a fully resolved airway up to generation 3, followed by a single flowpath model for generations $4-15$. Researchers at the University of Iowa [13] and the University of Auckland [14] have developed technologies for generating complete speciesspecific lung geometries based on space-filling algorithms for the airway branches. CFD simulations have been performed in which large portions of the airway region are fully resolved, and the lower airways are treated using a 1-D flow resistance model.

As suggested earlier, the current state of the art for CFD simulations is the use of partially resolved models of the lung airway, in which some branches are removed (truncated) from the airway tree, at multiple different levels of scale. This is necessitated by the fact that CFD simulations of the complete airway geometry will remain impractical for the foreseeable future [15]. The challenge is to provide physiologically realistic boundary conditions for these truncated geometries. The use of a serialized solution approach [7], [8] addresses this challenge by focusing in a sequential fashion on different regions of the airway, allowing a reasonable prescription of outlet boundary conditions a priori in each section, but eliminating the ability to perform fully coupled simulations of the entire airway tree. Current approaches for large-scale, fully coupled simulations include a priori pressure prescription at truncated flow outlets [10], a priori mass flow prescription at truncated flow outlets [11], [12], and coupling of truncated flow outlets to 1-D flow resistance models [9], [13].

An alternative to these methods has been recently proposed [15]. Dubbed the stochastic coupling approach, it is based on random mapping of flow information from resolved interior regions of the airway tree to unresolved (truncated) flow outlets. Consistency is maintained by ensuring that the mapping 


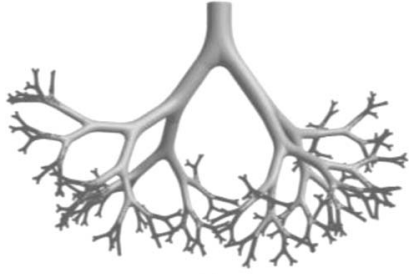

(a)

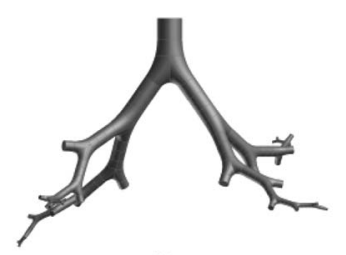

(b)

Fig. 1. Idealized eight-generation airway tree geometries based on symmetric Weibel [17] morphology. (a) Fully resolved model. (b) Partially resolved model.

occurs intragenerationally. The method allows fully coupled simulation over all morphological scales, eliminates the need for a priori prescription of boundary conditions at nonterminal outlets, and does not require coupling between 3-D CFD and 1-D flow resistance models. It was shown in [15] that the method could accurately approximate the flow in an idealized eight-generation bronchial tree [see Fig. 1(a)] using a significantly smaller geometry composed of only four resolved flow paths [see Fig. 1(b)]. The pressure drop was predicted by the reduced geometry model to within $1.5 \%$, and the average mass flow at the terminal outlets was predicted to within $2 \%$. The reduced geometry model shortened the overall simulation time and memory requirement by approximately one order of magnitude. Subsequent work showed that the method could also be used to accurately predict particle transport and deposition in similar idealized geometries [16].

The study presented here seeks to evaluate the feasibility of the stochastically coupled boundary condition method for use with physiologically realistic reduced geometry models. The test model is based on patient-specific CT-scan data of the extrathoracic region and a lower airway dataset available in the open literature [18]. In contrast to [15] and [16], the geometry considered here includes asymmetric branching angles, variability of intragenerational airway lengths and diameters, noncylindrical airways, and incomplete resolution of airway flowpaths to the terminal outlets. The model represents the entire conducting zone including orotracheal region, bronchi, and bronchioles, and extends to a maximum generation 18 of the airway tree. Airflow solutions are obtained for three steady inhalation flow rates, corresponding to realistic breathing rates for different levels of exertion in healthy adult human subjects. Results are evaluated based on the total pressure drop in the upper and lower airway regions, and are compared to available in vivo experimental data.

\section{LUNG GEOMETRY/MESH CONSTRUCTION}

In order to create an accurate oral cavity and throat model, the commercial software package Mimics (Materialise, Leuven,

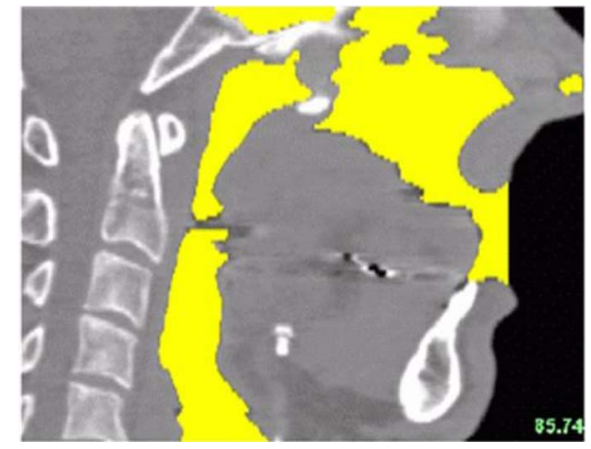

Fig. 2. Sagittal CT data plane of human nasopharyngeal passage segmented to isolate the air phase.

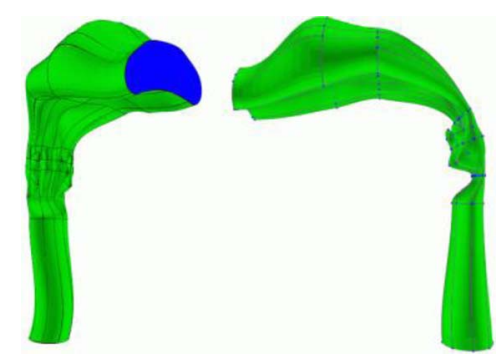

Fig. 3. Manually constructed notional model of a human oral cavity, larynx, and trachea inferred from CT data.

Belgium) was used to visualize and process anonymous CT data of human nasopharyngeal passages. Mimics allows the user to select regions of the body based on imaging intensity. In this case, the area with the lowest intensity (i.e., the air phase) was segmented to isolate the interior airways (see Fig. 2). Segmentation of the air phase at each imaging plane was stacked to define the upper airway volume.

The oral cavity and pharynx structures were manually constructed using SolidMesh, an in-house geometry and mesh generation tool, rather than automatically generated with the available Mimics tools. This was done for two reasons: 1) the CT data consisted of mostly nasal passages, whereas we desired a model with an oral cavity; and 2) the CT data were corrupt in the middle of the throat. The oral cavity was manually constructed based on the available contours in the CT scans and an approximate shape was used to fill the gap in the throat. The oropharynx model was connected to the remainder of the airways by an approximately cylindrically shaped trachea. Note that this airway section is not perfectly cylindrical because, in general, the trachea must curve outward to bypass the aorta. The final assembled oral cavity and throat model is shown in Fig. 3.

The lower bronchial and bronchiole airways were generated by randomly selecting airways from the human bronchial tree dataset of Schmidt et al. [18] (see Fig. 4). In particular, for each of the five lung lobes, four terminal airways were randomly selected and retained in the model. The final truncated bronchial tree consisted of 20 isolated terminal airways and was appended to the upper orotracheal geometry. Lower airway geometry generation, merging, scaling, and orientation were handled via custom methods available within the commercial 


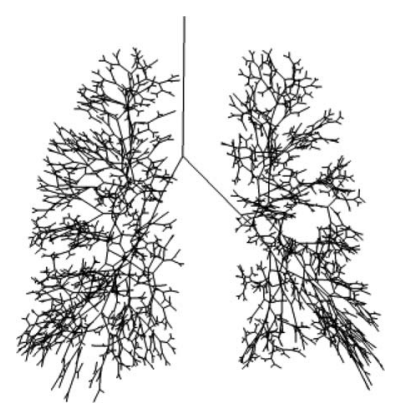

Fig. 4. Skeleton depiction of a human bronchial tree representation from Schmidt et al. [18].

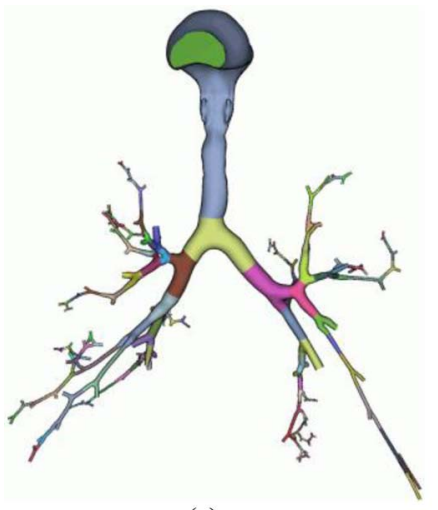

(a)

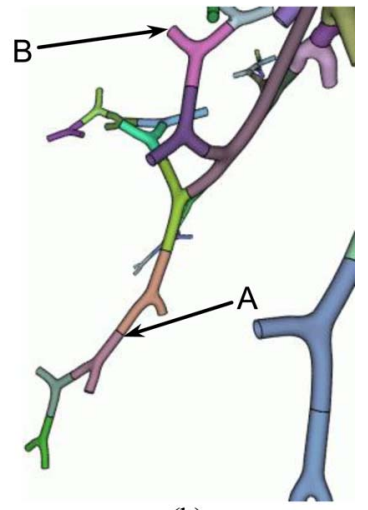

(b)
Fig. 5. Composite human lung model with 20 isolated airways modeled up to generation 18. (a) Complete model. (b) Detailed view of several terminal generations.

software package Discrete (Optimal LLC, Starkville, MS). The final composite lung geometry is shown in Fig. 5 and consisted of one flow inlet (the mouth) and 133 flow outlets. Six of the 133 flowpaths extended to generation 18, representing physical outlets for the reduced geometry model. All other outlets were considered to be truncated outlets. The generation 18 outlets had mean diameters of 500-600 $\mu \mathrm{m}$, in good agreement with documented airway sizes [2].

It should be noted that a fully resolved model up to generation 18 would necessarily contain $2^{17}$ individual airway segments in the lower airway region. In contrast, the truncated model used here contains 282 segments. It is, therefore, estimated that the truncated model reduces the computational expense by $99.8 \%$ (nearly three orders of magnitude) from a fully resolved 3D CFD simulation. The simulations reported in Gemci et al. [10] were also based on the Schmidt et al. [18] dataset, and contained 1453 flow segments. The geometry in the current work, therefore, represents a reduction of $80 \%$ in comparison to [10].

The mesh used in the simulations was similar to that in [16], and consisted of approximately $4.5 \mathrm{M}$ tetrahedral cells. For one test case, a refined mesh containing $36 \mathrm{M}$ cells was also run and showed only a small difference in pressure drop. The $4.5 \mathrm{M}$ cell case was, therefore, judged to be mesh independent and was used for all results shown below.

\section{NUMERICAL METHOD}

Simulations were performed using the commercial code Ansys FLUENT ${ }^{\circledR}$, v. 12.0 (Ansys, Inc., Canonsburg, PA). Results were obtained with second-order upwind discretization for convective terms, second-order central differencing for diffusive terms, the PRESTO! scheme for pressure discretization, and the SIMPLE algorithm for pressure-velocity coupling. Air properties were constant density $\left(\rho=1.225 \mathrm{~kg} / \mathrm{m}^{3}\right)$ and viscosity $\left(\mu=1.7894 \times 10^{-5} \mathrm{~kg} / \mathrm{m} \cdot \mathrm{s}\right)$. Steady inspiratory flow was modeled, with a uniform velocity applied at the mouth inlet, to produce volumetric flowrates $Q$ of 167,333 , and $500 \mathrm{~cm}^{3} / \mathrm{s}$. The pressure at each of the six generation 18 bronchiole outlets was set to a constant value of $0 \mathrm{~Pa}$ (gage). All other outlets were specified using the stochastic coupling boundary condition approach. Briefly, this approach randomly maps the pressure at resolved interior locations of the airway tree (e.g., location "A" in Fig. 5), to unresolved outlet locations within equivalent generations (e.g., location "B" in Fig. 5). Unresolved outlet pressures, therefore, converge toward constant values. The reader is referred to [15] and [16] for a more detailed description of the method. For comparison purposes, alternate test cases were run using a priori specification of constant pressure $(0 \mathrm{~Pa})$ at all flow outlets. The flow was assumed laminar throughout the domain. Although this may lead to some disagreement in the orotracheal region and the upper airways [19], laminar flow is considered sufficient to evaluate the feasibility of the boundary condition method. Laminar flow has been used in previous simulations similar to the ones reported here [9].

\section{RESULTS}

Predicted pressure contours on the airway wall are shown in Fig. 6 for the middle flow rate case $\left(Q=333 \mathrm{~cm}^{3} / \mathrm{s}\right)$. The figure shows results with the stochastic coupling (SCBC) method and the constant pressure outlet (PBC) method. For both, it can be seen that a significant portion of the pressure drop occurs in the orotracheal region, specifically across the oropharynx. The remainder of the pressure drop occurs in the lower airway region between the trachea and generation 18 .

It is apparent that the PBC method produces physiologically unrealistic results, as expected, with outlet pressures that are too low in the large truncated branches. In contrast, the SCBC method shows pressures at truncated outlets that are consistent with pressures at similar locations in fully resolved regions of the airway. Significantly, the airway pressure is only "fixed" at six of the 133 flow outlets, yet the results show qualitatively accurate distributions within the entire geometry.

Fig. 7 shows the pressure drop $\Delta P$ using both methods in the upper and lower airway regions. The upper airway, defined as the mouth, larynx, and trachea, is illustrated by the blue-gray region shown in Fig. 5(a). The lower airway is the remaining tracheobronchial tree. It is apparent from Fig. 7 that the pressure drop in the upper airway is equal regardless of which pressure boundary conditions are used. The lower airways, however, show considerable differences between the SCBC and PBC methods. The predicted resistance using the SCBC method ranges from $2.94 \mathrm{~cm} \mathrm{H}_{2} \mathrm{O} / \mathrm{L} / \mathrm{s}$ at $Q=167 \mathrm{~cm}^{3} / \mathrm{s}$ to $5.22 \mathrm{~cm} \mathrm{H}_{2} \mathrm{O} / \mathrm{L} / \mathrm{s}$ at 


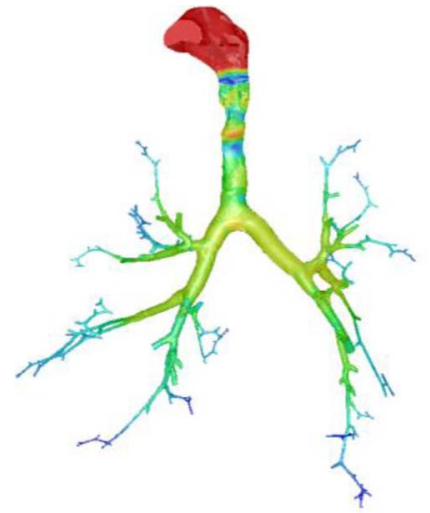

(a)

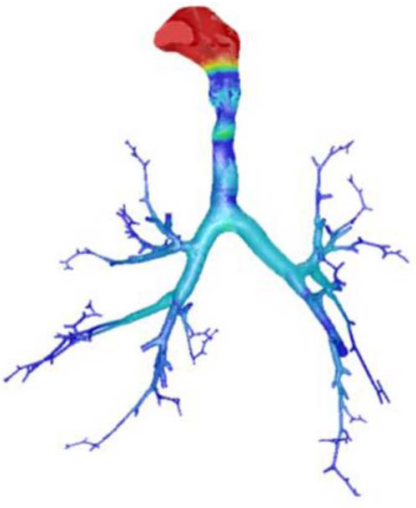

(b)
Fig. 6. Pressure distribution on airway wall for middle flow rate $(Q=$ $333 \mathrm{~cm}^{3} / \mathrm{s}$ ) case: (a) stochastically coupled outlet boundary conditions (SCBC); (b) a priori pressure outlet boundary conditions (PBC).

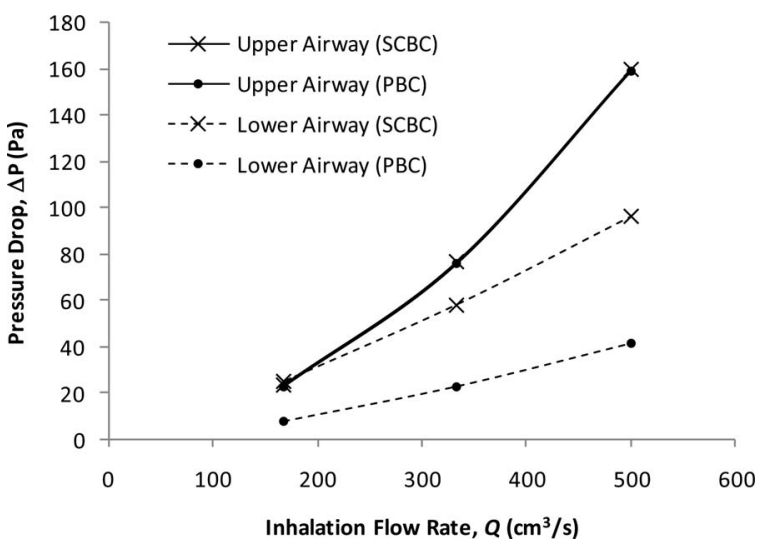

Fig. 7. Predicted pressure drop versus inhalation flow rate for cases with stochastically coupled boundary conditions (SCBC) and a priori pressure conditions (PBC).

$Q=500 \mathrm{~cm}^{3} / \mathrm{s}$. The results are in reasonable agreement with the in vivo lung flow data presented in [9] and [20], which reported a value of $3.6 \mathrm{~cm} \mathrm{H}_{2} \mathrm{O} / \mathrm{L} / \mathrm{s}$ for the total resistance of the lung airway. It is likely $\Delta P$ is slightly overpredicted in the lower airway due to the fact that several segments in the Schmidt dataset have exaggerated lengths (see Fig. 5) in comparison to morphometric data [2].

Further study is required to determine whether the SCBC method can accurately address regional variation in ventilation patterns arising from differences in tissue deformation during breathing and differences in distal airway paths, although the steady-state results presented here are encouraging.

\section{CONCLUSION}

The stochastic coupling boundary condition method documented previously [15], [16] has been extended to a physiologically realistic lung airway geometry, yielding pressure drop predictions in reasonable agreement with published in vivo data.
The method provides the potential for large-scale CFD simulations of the complete lung airway, with no need for sequential solution methods, nonphysical a priori boundary conditions, or coupling with 1-D resistance models. Future work will include extensive validation efforts and investigation of the effects of flow unsteadiness, turbulence, and regional, time-dependent variations in airway geometry.

\section{REFERENCES}

[1] C. Kleinstreuer and Z. Zhang, "Airflow and particle transport in the human respiratory system," Annu. Rev. Fluid Mech., vol. 42, pp. 301-334, 2010.

[2] H.-C. Yeh and G. M. Schum, "Models of human lung airways and their application to inhaled particle deposition," Bulletin Math. Biol., vol. 42, pp. 461-480, 1980.

[3] I. Balásházy, W. Hofmann, and T. Heistracher, "Computation of local enhancement factors for the quantification of particle deposition patterns in airway bifurcations," J. Aerosol Sci., vol. 30, pp. 185-203, 1999.

[4] Z. Zhang, C. Kleinstreuer, and C. S. Kim, "Cyclic micron-size particle inhalation and deposition in a triple bifurcation lung airway model," $J$. Aerosol Sci., vol. 33, pp. 257-281, 2002.

[5] Z. Zhang, C. Kleinstreuer, and C. S. Kim, "Gas-solid two-phase flow in a triple bifurcation lung airway model," Int. J. Multiphase Flow, vol. 28, pp. 1021-1046, 2002.

[6] B. Soni, C. Lindley, and D. Thompson, "The combined effects of nonplanarity and asymmetry on primary and secondary flows in the small bronchial tubes," Int. J. Numerical Methods Fluids, vol. 59, pp. 117-146, 2009.

[7] N. Nowak, P. P. Kadake, and A. V. Annapragada, "Computational fluid dynamics simulation of airflow and aerosol deposition in human lungs," Annals Biomed. Eng., vol. 31, pp. 374-390, 2003.

[8] Z. Zhang, C. Kleinstreuer, and C. S. Kim, "Airflow and nanoparticle deposition in a 16-generation tracheobronchial airway model," Ann. Biomed. Eng., vol. 36, pp. 2095-2110, 2008.

[9] B. Ma and K. R. Lutchen, "An anatomically based hybrid computational model of the human lung and its application to low frequency oscillatory mechanics," Ann. Biomed. Eng., vol. 34, pp. 1691-1704, 2006.

[10] T. Gemci, V. Ponyavin, Y. Chen, H. Chen, and R. Collins, "Computational model of airflow in upper 17 generations of human respiratory tract," $J$. Biomech., vol. 41, pp. 2047-2054, 2008.

[11] J. W. De Backer, W. G. Vos, S. C. Vinchurkar, R. Claes, A. Drollman, D. Wulfrank, P. M. Parizel, P. Germonpré, and W. De Backer, "Validation of computational fluid dynamics in CT-based airway models with SPECT/CT," Radiology, vol. 257, pp. 854-862, 2010.

[12] G. Tian, P. W. Longest, G. Su, and M. Hindle, "Characterization of respiratory drug delivery with enhanced condensational growth using an individual path model of the entire tracheobronchial airways," Ann. Biomed. Eng., vol. 39, pp. 1136-1153, 2011.

[13] C.-L. Lin, M. H. Tawhai, G. McLennan, and E. A. Hoffman, "Multiscale simulation of gas flow in subject-specific models of the human lung," IEEE Eng. Med. Biol. Mag., vol. 28. no. 3, pp. 25-33, May/Jun. 2009.

[14] K. S. Burrowes, E. A. Hoffman, and M. H. Tawhai, "Species-specific pulmonary arterial asymmetry determines species differences in regional pulmonary perfusion," Ann. Biomed. Eng., vol. 37, pp. 2497-2509, 2009.

[15] D. K. Walters and W. H. Luke, "A method for three-dimensional NavierStokes simulations of large-scale regions of the human lung airway," $J$. Fluids Eng., vol. 132, pp. 051101-1-051101-8, 2010.

[16] D. K. Walters and W. H. Luke, "Computational fluid dynamics simulations of particle deposition in large-scale, multigenerational lung models," $J$. Biomech. Eng., vol. 133, pp. 011003-1-011003-8, 2011.

[17] E. R. Weibel, Morphometry of the Human Lung. New York: Academic, 1963.

[18] A. Schmidt, S. Zidowitz, A. Kriete, T. Denhard, S. Krass, and H.-O. Peitgen, "A digital reference model of the human bronchial tree," Comput. Med. Imaging Graphics, vol. 28, pp. 203-211, 2004.

[19] P. W. Longest and S. Vinchurkar, "Validating CFD predictions of respiratory aerosol deposition: Effects of upstream transition and turbulence," $J$. Biomech., vol. 40, pp. 305-316, 2007.

[20] K. R. Lutchen, K. Yang, D. W. Kaczka, and B. Suki, "Optimal ventilation waveforms for estimating low-frequency respiratory impedance," J. Appl. Physiol., vol. 75, pp. 47-488, 1993. 